\title{
Homologous Recombination and Replication Fork Protection: BRCA2 and More!
}

\author{
WEIRAN FenG ${ }^{1,2}$ AND MARIA JASIN ${ }^{1,2}$ \\ ${ }^{1}$ Developmental Biology Program, Memorial Sloan Kettering Cancer Center, New York, New York 10065 \\ ${ }^{2}$ Louis V. Gerstner, Jr. Graduate School of Biomedical Sciences, Memorial Sloan Kettering \\ Cancer Center, New York, New York 10065 \\ Correspondence: m-jasin@ski.mskcc.org
}

\begin{abstract}
BRCA2 is a breast and ovarian tumor suppressor that guards against genome instability, a hallmark of cancer. Significant progress has been made in improving our understanding of BRCA2 function from biochemical, cellular, and mouse studies. The knowledge gained has been actively exploited to develop therapeutic strategies, including PARP inhibition, which has shown promising clinical outcomes. Recently, tremendous excitement has been generated by the findings of the roles of BRCA2 and other proteins in suppressing replication stress through homologous recombination and in the protection of stalled replication forks. Processes such as mitotic DNA synthesis and fork reversal have taken center stage in these studies. Here, we discuss our recent findings in the context of these advances.
\end{abstract}

BRCA2 is a well-known tumor suppressor that was identified more than two decades ago (Wooster et al. $1995)$ yet interest in this protein continues to grow. Monoallelic inheritance of a deleterious BRCA2 mutation confers up to a $70 \%$ risk for breast cancer and a $40 \%$ risk for ovarian cancer before age 70 (Antoniou et al. 2003), and a lower risk for other tumor types. Although retained in some cases (Maxwell et al. 2017), the wild-type allele is typically lost in tumor cells from $B R C A 2$ mutation carriers. Therefore, tumor formation is typically associated with a severe disruption in BRCA2 function. Somatic $B R C A 2$ mutations have also been identified more recently in several tumor types, including ovary (Pennington et al. 2014) and prostate (Robinson et al. 2015). Biallelic $B R C A 2$ germline mutations predispose to Fanconi anemia, a syndrome characterized by developmental defects and tumor susceptibility (D'Andrea 2010). In these cases, at least one of the BRCA2 alleles is expected to be hypomorphic, since complete loss of BRCA2 function causes embryonic lethality in mice (Evers and Jonkers 2006).

It is widely accepted that BRCA2 suppresses tumor formation by preventing genome instability, a hallmark of cancer (Hanahan and Weinberg 2011). Nevertheless, it is not fully understood how BRCA2 loss, or genome instability in general, promotes tumor formation. There is a paradox in that BRCA2 deficiency leads to cell lethality in mouse models (Patel et al. 1998; Evers and Jonkers 2006; Kuznetsov et al. 2008; Badie et al. 2010), rather than unrestrained proliferation as might be expected by loss of a tumor suppressor gene. BRCA2 has a wellestablished function in maintaining the integrity of the genome through its role in homologous recombination (HR). More recently, a related but separable function, replication fork protection (FP), has been discovered, and the relative contributions of these two pathways to genome integrity maintenance and cell viability are under active investigation. Here, we summarize the recent findings from several laboratories, including our own, on the function of BRCA2 with implications for cancer development and treatment.

\section{BRCA2, GENOME INTEGRITY MAINTENANCE, AND CANCER}

The role of BRCA2 in HR has been a subject of active investigation for many years (Prakash et al. 2015). HR repairs DNA lesions including DNA double-strand breaks (DSBs) using a homologous DNA sequence, typically the sister chromatid in mitotic cells. One critical step of HR is strand invasion, which primes subsequent DNA synthesis using the homologous sequence as a template, thereby ensuring an error-free repair outcome (Fig. 1). BRCA2 plays an essential role in this process by loading RAD51 recombinase onto single-stranded DNA formed at DSBs; the RAD51 nucleoprotein filaments then catalyze the subsequent strand invasion reaction (Jensen et al. 2010; Liu et al. 2010; Thorslund et al. 2010). Due to the HR deficiency, cells with impaired BRCA2 function are hypersensitive to cross-linking agents, such as cisplatin, and to poly(ADP-ribose) polymerase (PARP) inhibitors, which are being extensively explored as cancer therapeutics (Lord and Ashworth 2016).

FP is an additional BRCA2-mediated process that helps safeguard genomic integrity (Schlacher et al. 2011). Under replication stress, nascent DNA strands at stalled forks are susceptible to degradation by nucleases such as MRE11. BRCA2 prevents such nascent strand degrada-

(C) 2017 Feng and Jasin. This article is distributed under the terms of the Creative Commons Attribution License, which permits unrestricted reuse and redistribution provided that the original author and source are credited. 


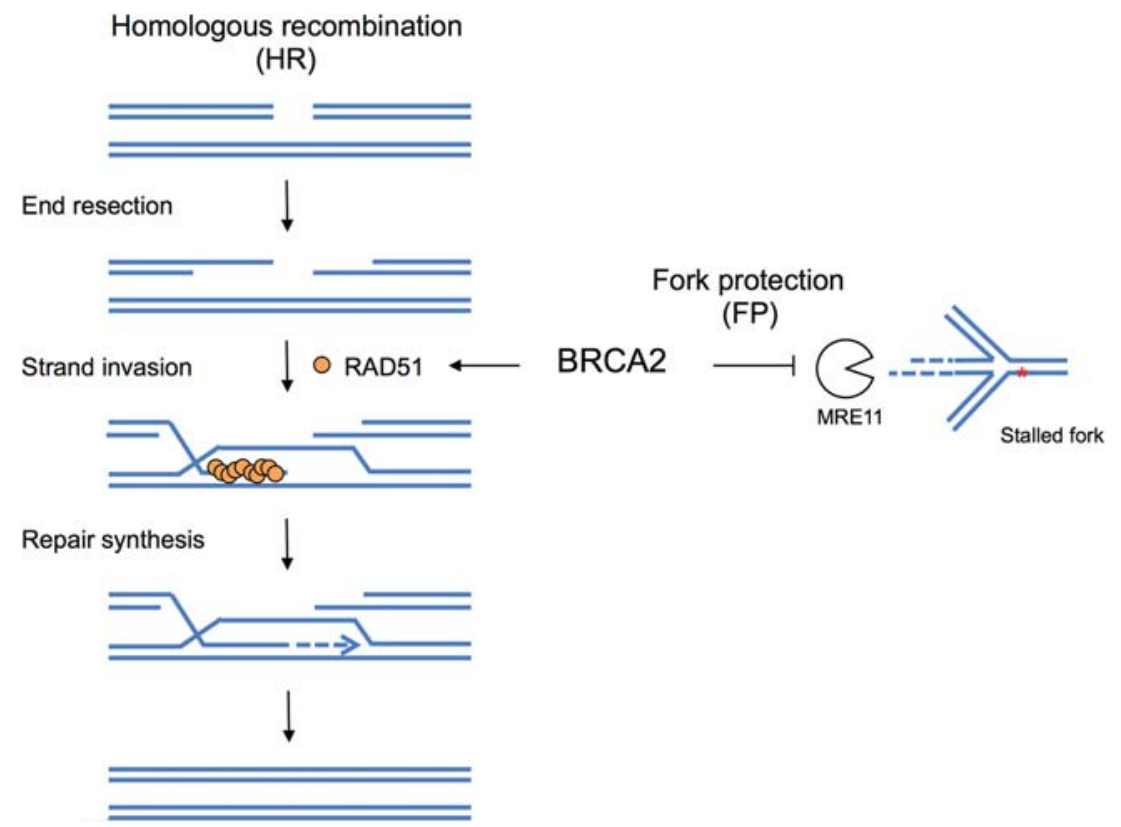

Figure 1. HR and FP functions of BRCA2 to protect genome integrity. (Left) HR repair of a DSB is initiated by resection of the break ends to generate 3' single-stranded DNA overhangs. Subsequent strand invasion into a homologous DNA is critical for repair DNA synthesis, which ultimately promotes an error-free repair outcome. Although RAD51 is crucial for strand invasion, BRCA2 plays an essential role by recruiting RAD51 onto the resected DNA. (Right) In the FP process, BRCA2 prevents the nascent strands of a stalled replication fork from being degraded by nucleases such as MRE11.

tion thereby protecting stalled forks (Fig. 1). In addition to BRCA2, other HR proteins, such as RAD51 itself, the breast and ovarian cancer suppressor BRCA1, and Fanconi anemia proteins also play important roles in the FP pathway (Schlacher et al. 2011, 2012). However, FP and HR are functionally separable, as evidenced by multiple approaches that specifically manipulate one process without affecting the other (Schlacher et al. 2011; Ding et al. 2016; Ray Chaudhuri et al. 2016; Dungrawala et al. 2017; Feng and Jasin 2017; Taglialatela et al. 2017). Of note, the role of RAD51 in FP seems to be more complicated than BRCA2: disruption of its function compromises FP in some studies (Schlacher et al. 2011; Kolinjivadi et al. 2017), but does not affect FP activity under other circumstances (Thangavel et al. 2015; Feng and Jasin 2017; Lemacon et al. 2017; Mijic et al. 2017). The seeming discrepancy can be attributed to an additional function of RAD51, that of fork reversal (Zellweger et al. 2015), which seems to be required for nascent strand degradation, a subject that is currently a focus of the field (discussed in detail below).

Since the initial identification of BRCA1 and BRCA2, a series of other HR factors, in particular PALB2, which bridges BRCA1 and BRCA2, and RAD51 paralogs RAD51C and RAD51D have been identified as tumor suppressors (Erkko et al. 2007; Rahman et al. 2007; Tischkowitz et al. 2007; Meindl et al. 2010; Loveday et al. 2011). Where tested, these proteins also have roles in FP (Fig. 3; Somyajit et al. 2015). Similar to BRCA1 and BRCA2 deficiency, disruption of these proteins also causes sensitivity to cross-linking agents and PARP inhibitors (Prakash et al. 2015). BRCA1/2 mutant cancers exhibit a particular pattern of base substitutions and genome rearrangements - that is, "mutational signatures" (Nik-Zainal et al. 2016; Polak et al. 2017). A computational model based on these mutational signatures has been used to predict additional patient tumors with HR-deficiency beyond those containing mutations in known HR genes, thus expanding the pool of cancers that may respond to platinum or PARP inhibitor therapy (Davies et al. 2017).

Despite encouraging success in the clinic, resistance to platinum drugs and PARP inhibitor therapy can eventually be acquired by tumors. Secondary mutations in the mutated HR genes are frequently observed that reestablish the reading frame and, when checked, restore protein function (Edwards et al. 2008; Sakai et al. 2008; Norquist et al. 2011; Kondrashova et al. 2017; Chen et al. 2018). Remarkably, in a recent study of circulating tumor DNA from prostate cancer patients, 34 secondary mutations were identified in a single patient that restored the BRCA2 reading frame to confer therapy resistance (Quigley et al. 2017). HR restoration is usually considered to be the underlying mechanism, although FP is likely to be restored as well in most instances. Interestingly, FP restoration has been associated with chemoresistance in experimental models, even without restoration of HR (Ray Chaudhuri et al. 2016). Validating the causal relationship between FP and therapy resistance in patients will be critical to understand the clinical relevance of FP.

\section{CONSEQUENCES OF BRCA2 DEFICIENCY}

One puzzle in the field is that, while predisposing to cancer, BRCA2 deficiency paradoxically leads to invia- 
bility in mice, both in embryos and in cells (Patel et al. 1998; Evers and Jonkers 2006; Kuznetsov et al. 2008; Badie et al. 2010). Therefore, a gap in our understanding needs to be bridged between the immediate consequence of cell inviability and the long-term tumor susceptibility from BRCA2 deficiency. In addition, while BRCA2 loss is expected to impair HR in all tissues (Kass et al. 2016), it predominantly predisposes to cancer in the breast and ovary. Resolution of these paradoxes requires a cellular model from a disease-relevant tissue, such as human mammary epithelial cells.

We recently set out to approach these questions by generating BRCA2 conditional models in a nontransformed human mammary epithelial cell line with a relatively stable genome (MCF10A; [Soule et al. 1990; Cowell et al. 2005]). This study reveals that BRCA2-deficiencytriggered cell lethality is conserved in these relatively normal human mammary cells (Feng and Jasin 2017). BRCA2 deficiency leads to cell cycle arrest in $\mathrm{G}_{1}$, a surprising result considering that BRCA2 functions in genome integrity maintenance pathways that are active in $\mathrm{S}$ and $\mathrm{G}_{2}$. To reconcile these seemingly counterintuitive results, we traced the source of DNA lesions that occur upon BRCA2 loss. We found that BRCA2 inactivation leads to DNA under replication, which in turn causes abnormalities during mitosis and 53BP1 nuclear body formation in the subsequent $\mathrm{G}_{1}$ phase associated with a p53-dependent cell cycle arrest (Fig. 2; Feng and Jasin 2017). Independent results from other groups are in agreement with this model (Lai et al. 2017; Schoonen et al. 2017). Notably, while mitotic abnormalities have previously been associated with BRCA2 deficiency (Tutt et al. 1999; Daniels et al. 2004; Laulier et al. 2011; Choi et al. 2012; Mondal et al. 2012), we establish that it is the premitotic stresses from the $S$ and $G_{2}$ phases that cause the subsequent aberrations (chromosome missegregation, 53BP1 nuclear body formation), given that delaying mitotic entry abrogates these abnormalities (Feng and Jasin 2017).

What is the cause of DNA under replication? BRCA2deficient cells accumulate single-stranded DNA lesions in $\mathrm{G}_{2}$ (Feng and Jasin 2017). Fork reversal, hyperresection at DNA breaks (Feng and Jasin 2017), and single-stranded DNA formed at or behind the forks (Kolinjivadi et al. 2017) can all contribute to these lesions. We propose that these persistent, unrepaired DNA lesions prevent timely completion of DNA replication. Thus, the sequelae of BRCA2 deficiency can be traced from $\mathrm{S} / \mathrm{G}_{2}$ DNA lesions as the source of the $G_{1}$ arrest to cell inviability as the consequence (Fig. 2; Feng and Jasin 2017).

\section{INVOLVEMENT OF p53 AFTER BRCA2 LOSS}

The above model predicts that the lethal phenotypes of BRCA2 disruption may be mitigated by inactivation of cell cycle arrest processes. The p53 pathway is induced upon BRCA2 loss and is responsible for the subsequent $\mathrm{G}_{1}$ arrest. Indeed, p53 loss partially restores cell proliferation to BRCA2-deficient cells (Feng and Jasin 2017). These observations provide insight into the frequent asso-

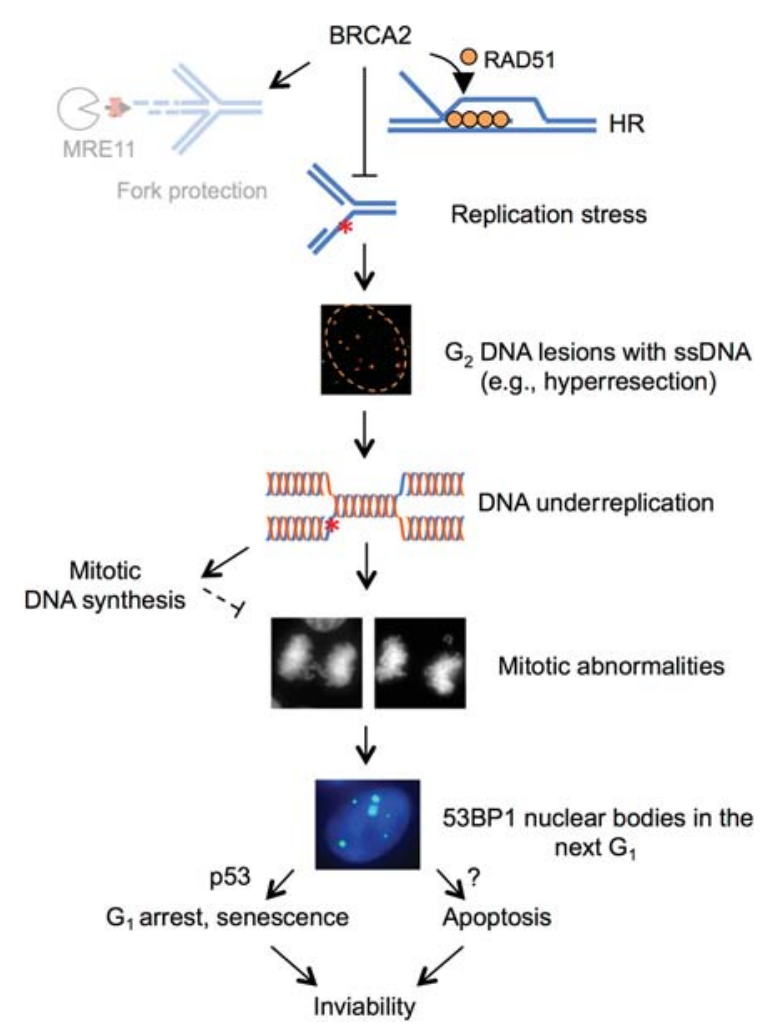

Figure 2. Consequences of BRCA2 deficiency in multiple cell cycle phases. As proposed in our recent study using MCF10A cells (Feng and Jasin 2017), even in unperturbed situations, BRCA2 deficiency causes replication stress that compromises the timely completion of DNA replication. The resulting underreplicated DNA leads to $\mathrm{G}_{2}$ DNA lesions and single-stranded DNA (ssDNA) formation, which in turn leads to abnormalities in mitosis and 53BP1 nuclear body formation in the subsequent $G_{1}$ phase. Such $\mathrm{G}_{1}$ lesions trigger $\mathrm{p} 53$-dependent $\mathrm{G}_{1}$ arrest and cellular senescence as well as p53-independent apoptosis, resulting in cell inviability. At the functional level, suppression of replication stress is primarily mediated by the HR, rather than the FP, activity of BRCA2. (Reprinted from Feng and Jasin 2017 under a Creative Commons license http://creativecommons.org/licenses/by/4.0/.)

ciation of TP53 mutations with BRCA2-mutated cancers (Ramus et al. 1999; Greenblatt et al. 2001; Roy et al. 2011), which parallels results from mouse systems (Jonkers et al. 2001; Bouwman et al. 2010). However, p53 inactivation only partially rescues MCF10A cells (Feng and Jasin 2017). While senescence and $G_{1}$ arrest are substantially abrogated by p53 loss, apoptosis is not (Feng and Jasin 2017), indicating that a p53-independent apoptotic pathway prevents full rescue of viability in the absence of BRCA2. One possibility is the involvement of other p53 family members (i.e., p63 and p73), which play similar roles to p53 in multiple processes, including apoptosis, and have been shown to act redundantly in some contexts (Dotsch et al. 2010; Wang et al. 2017). Therefore, multiple pathways, both p53-dependent and -independent, work together to ultimately result in inviability of BRCA2-deficient cells (Figs. 2, 5A).

p53 pathway activation in $\mathrm{G}_{1}$ could occur through several nonmutually exclusive ways. First, 53BP1 directly interacts with $\mathrm{p} 53$ and regulates p53-dependent $\mathrm{G}_{1}$ check- 
point arrest (Iwabuchi et al. 1994; Cuella-Martin et al. 2016). Therefore, p53 can be activated as a direct response to $53 \mathrm{BP} 1$ nuclear bodies. However, this interaction may not be the sole explanation for p53 activation, given that 53BP1 inactivation neither restores viability nor diminishes p53 induction in BRCA2-deficient mouse cells (Bouwman et al. 2010). Second, 53BP1 nuclear bodies mark DNA breaks due to improper resolution of underreplicated DNA during mitosis (Lukas et al. 2011; Naim et al. 2013; Ying et al. 2013); p53 activation can thus be triggered as a downstream event of DNA damage signaling. Consistent with this notion, a number of DNA damage response proteins reside in 53BP1 nuclear bodies (Lukas et al. 2011). In addition to 53BP1-related mechanisms, p53-dependent $\mathrm{G}_{1}$ arrest may also be activated by mitotic errors independently of DNA damage (Kuffer et al. 2013; Ganem et al. 2014; Pedersen et al. 2016). Collectively, we propose that the replication stress in BRCA2-deficient cells leads to abnormalities in the subsequent $\mathrm{M}$ and $\mathrm{G}_{1}$ phases, which in turn trigger apoptosis together with p53-dependent $\mathrm{G}_{1}$ arrest and cellular senescence (Fig. 2).

\section{FORK REVERSAL AND NASCENT STRAND DEGRADATION}

Unlike other canonical HR factors such as BRCA2, RAD51 plays a more complex role during FP. Manipulating RAD51 filament stability itself clearly impacts FP: Disrupting RAD51 filaments in wild-type cells, by overexpressing a BRC repeat from BRCA2, impairs FP, while stabilizing the filament, by expressing RAD51 K133R, can restore FP in deficient cells (Schlacher et al. 2011, 2012). Paradoxically, depletion of RAD51 itself, however, does not affect FP (Thangavel et al. 2015; Feng and Jasin 2017). Emerging evidence suggests that the resolution of this conundrum lies in the process of fork reversal that precedes nascent strand degradation (Fig. 3A). Fork rever-
A
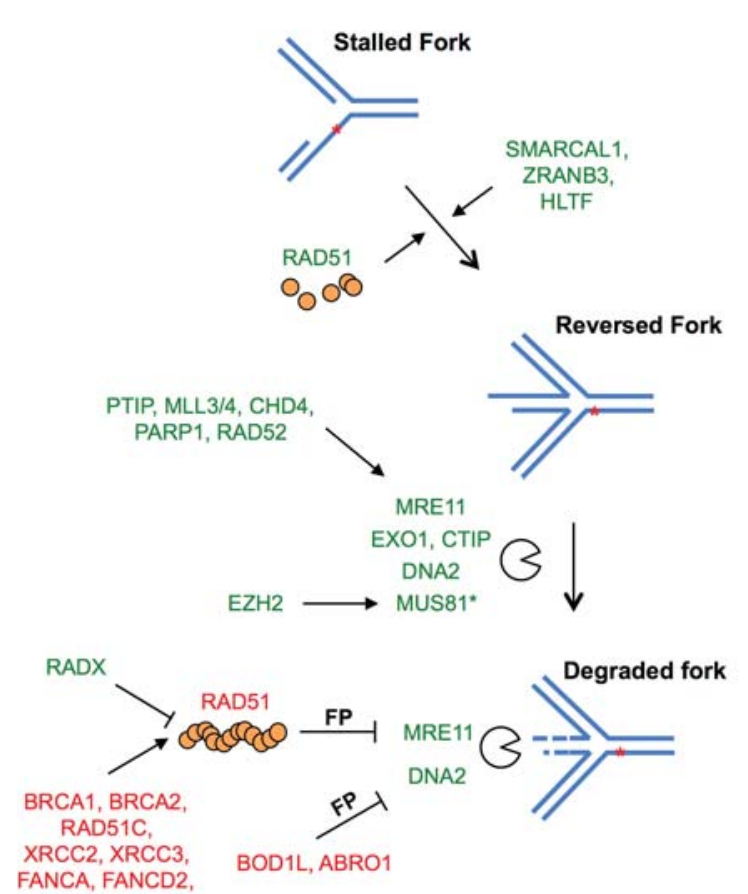

B
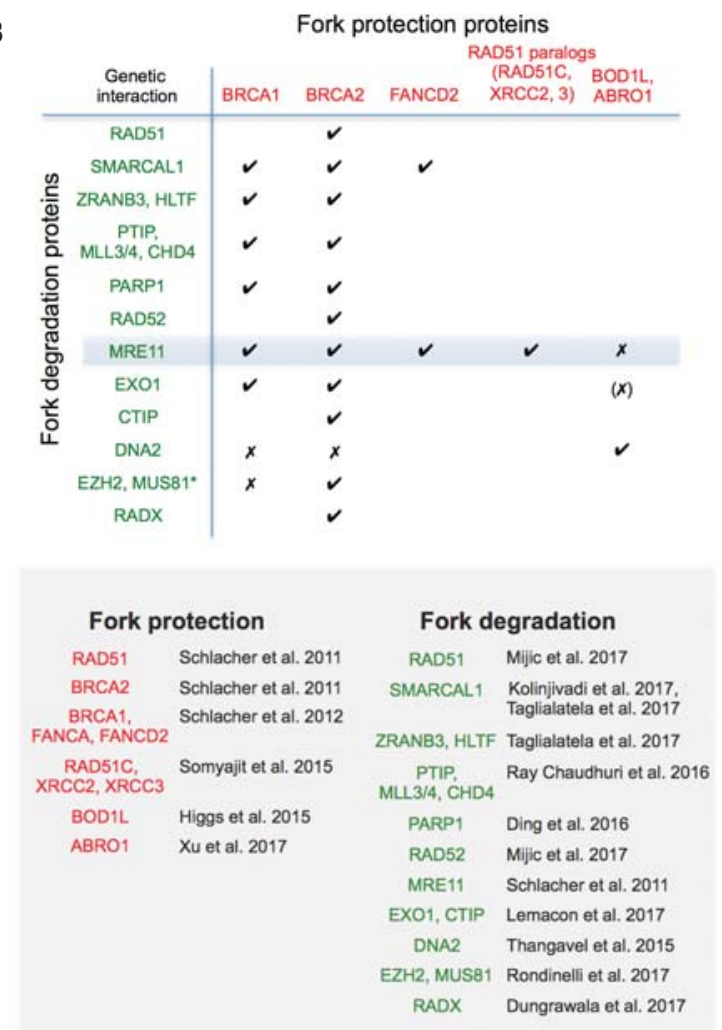

Figure 3. Mechanisms of fork degradation and protection. (A) Fork degradation occurs on a reversed fork. Reversal of stalled replication forks is promoted by RAD51 recombinase and DNA translocases (SMARCAL1, ZRANB3, HLTF). In the absence of FP factors like BRCA2, MRE11 and other nucleases can lead to fork degradation. Different pathways regulate MRE11 and MUS81 recruitment to stalled forks. MRE11 recruitment is promoted by a number of proteins, including PARP1 and the PTIP-MLL3/4 axis, whereas MUS81 recruitment relies on chromatin modifier EZH2. Prevention of MRE11-mediated fork degradation requires RAD51, which is facilitated by HR-Fanconi anemia proteins but antagonized by RADX. Thus, RAD51 both promotes and prevents fork degradation, in particular, at the steps of fork reversal and through the formation of stabilized filaments, respectively. Prevention of DNA2-mediated fork degradation involves BOD1L and ABRO1. Proteins that contribute to FP and degradation are labeled with a red and green color, respectively. Asterisk, MUS81's role in fork degradation is not always observed (discussed in the text). (B) Summary of the reported genetic interactions between FP proteins and proteins that directly or indirectly promote fork degradation, simplified here as "fork degradation proteins." A checkmark indicates that the absence of a FP protein leads to nascent strand degradation involving the corresponding fork degradation protein. An $x$ indicates that evidence exists that a given fork degradation protein is not responsible for fork degradation in the absence of the corresponding FP protein. $(x)$ indicates that EXO1 was not tested for BOD1L. Citations of proteins involved in FP and degradation are listed. 
sal refers to the remodeling of replication forks into a fourway junction structure, a process that ensures proper resumption of replication observed in a variety of species/ cell types including mammalian cells (Neelsen and Lopes 2015; Berti and Vindigni 2016; Quinet et al. 2017). Surprisingly, RAD51 is critical for reversing forks, but BRCA2 does not seem to play a role in this process (Zellweger et al. 2015; Mijic et al. 2017). Therefore, the fork reversal activity of RAD51 is distinguishable from its strand invasion function during the canonical HR process.

Reversed forks have emerged as the entry point for subsequent nascent strand degradation (Kolinjivadi et al. 2017; Lemacon et al. 2017; Mijic et al. 2017; Taglialatela et al. 2017). Indeed, RAD51 depletion in BRCA2-deficient cells precludes nascent strand degradation (Lemacon et al. 2017; Mijic et al. 2017), which we also observe in our system (Feng and Jasin 2017). Moreover, disruption of DNA translocases that have established fork reversal activity, such as SMARCAL1 (Bétous et al. 2012, 2013), ZRANB3 (Ciccia et al. 2012), and HLTF (Kile et al. 2015), also restores FP to BRCA2-deficient cells or BRCA2-depleted Xenopus egg extracts (Kolinjivadi et al. 2017; Lemacon et al. 2017; Mijic et al. 2017; Taglialatela et al. 2017). Together, these recent studies converge on a two-step model in which stalled replication forks are first reversed by RAD51 and DNA translocases, which are then vulnerable to nascent strand degradation, a step antagonized by the FP process mediated by BRCA2-dependent RAD51 filament formation/stabilization (Fig. 3A).

The fork reversal and protection activities of RAD51 prove to be functionally separable, as evidenced by the RAD51 T131P mutant expressed in a patient-derived cell line (Wang et al. 2015), which is proficient at fork reversal, but impaired in FP (Mijic et al. 2017).

The FP defect in these cells is presumably due to a failure to form stable RAD51 filaments (Wang et al. 2015), consistent with our previous findings using a BRCA2 separation of function mutant (Schlacher et al. 2011). Whether RAD51 has intrinsic fork reversal activity or promotes the activity of a translocase is not clear. Taken together, the ability of RAD51 to act at distinct biochemical steps may explain the diverse outcomes when RAD51 is perturbed in different ways.

\section{NUCLEASES INVOLVED IN NASCENT STRAND DEGRADATION}

Along with requiring a reversed fork as a substrate, nascent strand degradation involves multiple nucleases and epigenetic control. MRE11 was the first nuclease characterized to be involved in the resection of stalled forks in BRCA/FA-deficient backgrounds and remains the gold standard for analyzing FP pathways (Schlacher et al. 2011, 2012). MRE11 recruitment to stalled replication forks is mediated by histone $\mathrm{H} 3$ lysine 4 methyltransferases MLL3 and MLL4 (MLL3/4) and interacting protein PTIP (Ray Chaudhuri et al. 2016) and also relies on a continuously expanding list of proteins, such as PARP1 (Ding et al. 2016), CHD4 (Ray Chaudhuri et al.
2016), and RAD52 (Fig. 3A; Mijic et al. 2017). Either directly inhibiting MRE11 activity with small molecules or disrupting its recruitment by depleting any of these proteins restores FP to BRCA2- (and where tested BRCA1-) deficient cells (Fig. 3B), underscoring the critical role of MRE11 in nascent strand degradation.

In addition to MRE11, resection enzymes EXO1 and CTIP also independently mediate nascent strand degradation in BRCA1- and BRCA2-deficient cells (Fig. 3; Lemacon et al. 2017). In contrast, another resection nuclease DNA2 does not play a major role in these mutants (Thangavel et al. 2015; Ray Chaudhuri et al. 2016), although it has been shown to play a role in other contexts (i.e., with deficiencies of the FP factors BOD1L and ABRO1 [Fig. 3]). ABRO1 protects stalled forks independent of RAD51 (Xu et al. 2017a); while BOD1L promotes RAD51 chromatin loading (Higgs et al. 2015), whether stabilized RAD51 filaments are required in the absence of BOD1L remains to be tested. DNA2 also degrades stalled forks even in wild-type U2OS cells under prolonged replication stress resulting from hydroxyurea treatment, while MRE11, EXO1, and CTIP are not involved in this process (Thangavel et al. 2015). Therefore, multiple nucleolytic pathways are differentially activated in response to varying stresses and genetic perturbations.

The structure-specific nuclease MUS81 has a more complex involvement in the FP pathway. Parallel to the MLL3/4-PTIP recruitment path for MRE11 is the recently described EZH2-MUS81 axis (Fig. 3A), which provides one context in considering the role of MUS81. EZH2 is recruited to stalled forks and mediates methylation of histone $\mathrm{H} 3$ at lysine 27, and MUS81 is able to interact with this histone modification.

Perturbation of either EZH2 or MUS81 restores FP to BRCA2-defective cells (Rondinelli et al. 2017). This discovery further extends the scope of nucleases and epigenetic alterations at the fork that promote nascent strand degradation, although it remains to be determined whether MUS81 nuclease activity is directly involved. Interestingly, the EZH2-MUS81 axis only operates in BRCA2-, but not BRCA1-, deficient backgrounds (Fig. 3B; Rondinelli et al. 2017), indicating that the FP functions of BRCA1 and BRCA2 are separable. Accordingly, MUS81 chromatin recruitment is induced upon loss of BRCA2 (Bhowmick et al. 2016; Lai et al. 2017; Lemacon et al. 2017; Rondinelli et al. 2017), but not BRCA1 (Lemacon et al. 2017; Xu et al. 2017b). It is well appreciated that BRCA1 acts at a distinct step of HR, upstream of BRCA2 (Chen et al. 2018), but thus far, the biology behind their different roles in FP remains unclear and warrants future investigation.

While providing a risk to genome integrity, one potentially positive role for nascent strand degradation is to promote fork restart. BRCA2-deficient cells undergo extensive fork degradation but maintain normal fork restart activity, at least in some contexts (Schlacher et al. 2011). Two recent studies reported that MUS81 nuclease supports fork restart in the absence of BRCA2 (Lemacon et al. 2017; Rondinelli et al. 2017). MUS81 acts by cleaving degraded forks to mediate fork restart, a conclusion 
that is supported by the observation that MUS81 depletion in BRCA2-deficient cells leads to fewer DSBs and a concomitant increase of reversed forks, especially those with a single-stranded arm, indicative of extensive fork resection (Lemacon et al. 2017). Consistent with this, MUS81 depletion did not rescue FP in the BRCA2-deficient U2OS cells and in fact the cells are sensitized to hydroxyurea (Lemacon et al. 2017), in line with a synthetic lethal interaction between the two genes observed in another study (Lai et al. 2017).

However, Rondinelli et al., using a panel of other cell lines, has argued for a synthetic viable, rather than synthetic lethal interaction: MUS81 disruption restores FP upon BRCA2 deficiency to confer resistance to PARP inhibition (Rondinelli et al. 2017), although this was not observed by Lemacon et al. (2017). The discrepant roles of MUS81 in fork degradation could be related to its ability to process diverse types of substrates (Dehe and Gaillard 2017).

\section{CONTRIBUTIONS OF HR AND FP TO CELL FITNESS}

One key mechanistic question related to BRCA2 function is how it supports cell proliferation. HR factors like BRCA1 and BRCA2 are essential for embryonic cell survival, which has implicated $\mathrm{HR}$ as an essential process in mammalian cells (Moynahan and Jasin 2010). More recently, however, FP in the absence of HR has been reported to sustain viability of BRCA2-deficient cells, both mouse embryonic stem (ES) cells and tumor cells subjected to chemotherapy, arguing that HR may not be essential in these contexts (Ding et al. 2016; Ray Chaudhuri et al. 2016). Subsequent studies have also observed improved cell fitness (viability, chemoresistance) when nascent strand degradation is prevented, by suppressing either fork reversal (Taglialatela et al. 2017) or the RAD51 antagonist RADX (Dungrawala et al. 2017).

However, the correlation between FP and cell fitness does not always hold true. For example, cells with a mutation at the BRCA2 S3291 residue are severely disrupted in FP, but remain proficient in both unperturbed survival and preventing genome instability under PARP inhibitor/ platinum treatment, presumably because HR is intact (Schlacher et al. 2011; Feng and Jasin 2017). In fact, fully restoring FP to BRCA2-deficient cells by perturbing different proteins (ZRANB3, MUS81, RADX) (Fig. 3) varies dramatically in the extent to which it leads to acquired chemoresistance, ranging from no resistance (Lemacon et al. 2017; Mijic et al. 2017) to partial or substantial resistance (Dungrawala et al. 2017; Rondinelli et al. 2017). Different cellular contexts could contribute to the varying experimental outcomes. Therefore, it is critical to determine the functional relationship between HR and FP for viability of normal human cells, particularly in BRCA1/2-relevant mammary cells.

To approach this question, we generated three independent separation-of-function systems in MCF10A cells (Fig. 4). FP, but not HR, is selectively disrupted through expression of a mutant BRCA2 peptide (BRCA2 S3291E)

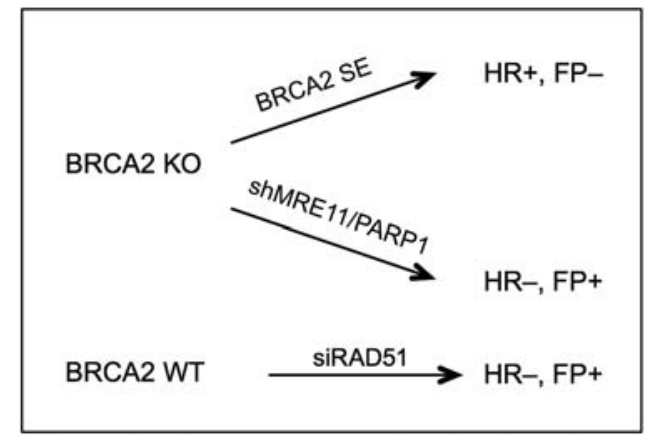

Figure 4. Separation-of-function systems to study HR and FP functions. Three separation-of-function systems to dissect the individual contributions of HR and FP pathways have been described (Feng and Jasin 2017).

or restored by MRE11 or PARP1 ablation in BRCA2deficent cells (Feng and Jasin 2017). In the third system, HR, but not FP, is selectively impaired by RAD51 depletion in BRCA2 wild-type cells (Feng and Jasin 2017). All three systems unambiguously show that HR, but not FP is critical to suppress replication stress and support cell viability in this nontransformed human mammary cell line (Fig. 2; Feng and Jasin 2017).

Thus, these two pathways, HR and FP, are differentially required for cell viability and genome integrity in different cell lines. How easy it is to meet the survival threshold, and thereby dictate the outcome of pathway restoration, may depend on the cellular context (Fig. 5). For example, the p53 pathway is compromised in mouse ES cells (Aladjem et al. 1998), such that these cells may have a higher tolerance to DNA damage; accordingly, preventing nascent strand degradation alone may be sufficient for their survival. Similarly, having gone through the stresses of BRCA2 loss, tumor cells have evolved to tolerate HR loss, such that FP restoration alone may suffice to reduce genome instability and thereby confer chemoresistance (Fig. 5). In agreement with a context-dependent pathway requirement, SMARCAL1 inactivation, preventing fork degradation, alleviates sensitivity to PARP inhibitors and cisplatin of BRCA1-depleted breast cancer MDA-MB-231 cells, but not MCF10A cells (Taglialatela et al. 2017). Future studies are needed to understand the relationship between these pathways and various cellular milieus.

\section{IMPLICATIONS FOR THERAPY}

More than a decade ago, seminal work on PARP inhibition opened a new avenue of exploiting synthetic lethality to target cancers with BRCA1/2 deficiency (Bryant et al. 2005; Farmer et al. 2005). PARP inhibitors have clearly shown promise with FDA approval for ovarian and, more recently, breast cancer treatment. Underlying mechanisms for the hypersensitivity of BRCA1/2-mutant tumor cells to PARP inhibition include accumulation of single-strand breaks and especially trapping of PARP protein on DNA to impede replication, both of which lead to lesions that require HR to be repaired (Lord and Ashworth 
A

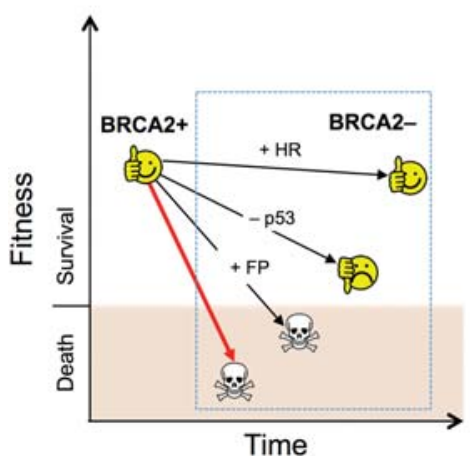

B

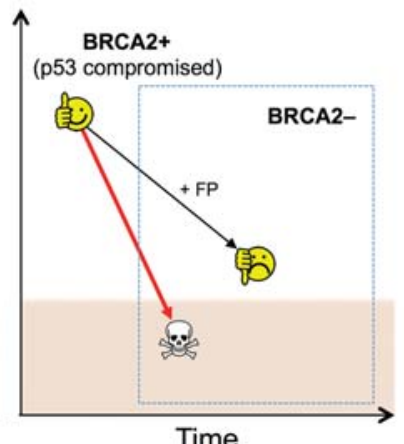

C Tumor cells

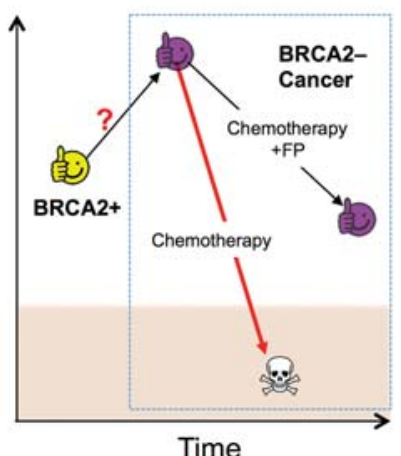

Figure 5. Context-dependent requirement for HR versus FP for cell survival. Differential requirements for HR and FP are observed to reach the threshold of cell viability in different contexts of BRCA2 deficiency, as highlighted in the dashed blue boxes. BRCA2 loss alone (untreated or with chemotherapy) (red arrows) is shown as a baseline for comparison to BRCA2 loss with the indicated additional genetic alterations (black arrows). (A) Nontransformed mammary epithelial cells MCF10A do not survive BRCA2 loss. Restoration of $\mathrm{HR}$, but not FP, re-establishes cell viability. p53 loss fails to suppress apoptosis and only partially rescues cell proliferation. $(B)$ In mouse ES cells, FP restoration is sufficient to rescue cell survival after BRCA2 loss. These cells may have a higher tolerance to DNA damage than MCF10A cells due to a compromised p53 response. $(C)$ In BRCA2-deficient tumor cells, FP is also sufficient to overcome the viability threshold to confer chemoresistance. Presumably, these cells have evolved to survive BRCA2 loss and aberrantly proliferate (purple face) and thus may have a higher tolerance to DNA damage.

2017). Impaired mitotic progression may also promote PARP inhibition-associated cytotoxicity (Schoonen et al. 2017).

Despite the promise, PARP inhibitor therapies are challenged by tumor relapse with acquired resistance (Lord and Ashworth 2017). Thus, novel independent strategies to target $B R C A 1 / 2$ cancers may complement current therapies. Exploiting an increasing dependence of HR-deficient cells on minor DSB repair pathways has led to the discovery of novel targets for synthetic lethality: POL $\theta$, which plays a role in microhomology-mediated end joining (Ceccaldi et al. 2015; Mateos-Gomez et al. 2015), and RAD52, a nonessential HR factor which becomes essential for the residual HR in BRCA1/2-deficient cells (Feng et al. 2011; Lok et al. 2013). More recently, another strategy to target has been suggested that involves the phenomenon of mitotic DNA synthesis. Even under unperturbed conditions, BRCA2 prevents DNA under replication (Feng and Jasin 2017; Lai et al. 2017), such that in the absence of BRCA2 mitotic DNA synthesis is activated (Feng and Jasin 2017; Lai et al. 2017), presumably as a last resort to complete DNA replication and prevent mitotic catastrophe (Minocherhomji et al. 2015; Bhowmick et al. 2016). A potential target in this pathway is RAD52, which is proposed to help prime mitotic DNA synthesis (Bhowmick et al. 2016). Other mitotic DNA synthesis components include MUS81 or SLX4, (Minocherhomji et al. 2015), loss of which impairs survival of BRCA2deficient cancer cells (Lai et al. 2017). Interestingly, in contrast to the case of otherwise unperturbed cell proliferation, upon PARP inhibition MUS81 depletion paradoxically improves the viability of BRCA2-deficient cells, as discussed above (Rondinelli et al. 2017). Given that the critical pathways for cell survival may vary under different stresses, the importance of the accurate design of therapy strategies cannot be overstated.

\section{CONCLUSION}

Significant progress has been made in advancing our understanding of BRCA2 functions, in particular, the interplay between replication and repair, therapy design, and resistance mechanisms. Despite the recent progress, we are only starting to learn the functional relationship between HR and FP, and processes like fork reversal and mitotic DNA synthesis. Also remaining largely unexplored are the complexity of the differential contributions of these pathways in diverse contexts and its possible implications in different biological processes (i.e., development, tumor formation, therapy response, and resistance acquisition). Furthermore, the additional tumor suppressive barriers that impede cell survival after BRCA2 loss are not fully understood. Future studies are needed to provide insight into these aspects to improve the picture of BRCA2 biology as well as strengthen cancer therapy.

\section{ACKNOWLEDGMENTS}

We thank members of the Jasin laboratory for discussions and suggestions. W.F. was supported by an Olayan Fellowship. The Jasin laboratory is supported by MSK Cancer Center Support Grant/Core Grant P30 CA008748, a Geoffrey Beene Cancer Research Center Grant, and National Institutes of Health (NIH) grant R01 CA185660.

\section{REFERENCES}

Aladjem MI, Spike BT, Rodewald LW, Hope TJ, Klemm M, Jaenisch R, Wahl GM. 1998. ES cells do not activate p53dependent stress responses and undergo p53- independent apoptosis in response to DNA damage. Curr Biol 8: 145-155.

Antoniou A, Pharoah PDP, Narod S, Risch HA, Eyfjord JE, Hopper JL, Loman N, Olsson H, Johannsson O, Borg A,, 
et al. 2003. Average risks of breast and ovarian cancer associated with BRCA1 or BRCA2 mutations detected in case series unselected for family history: A combined analysis of 22 studies. Am J Hum Genet 72: 1117-1130.

Badie S, Escandell JM, Bouwman P, Carlos AR, Thanasoula M, Gallardo MM, Suram A, Jaco I, Benitez J, Herbig U, et al. 2010. BRCA2 acts as a RAD51 loader to facilitate telomere replication and capping. Nat Struct Mol Biol 17: 1461-1469.

Berti M, Vindigni A. 2016. Replication stress: Getting back on track. Nat Struct Mol Biol 23: 103-109.

Bétous R, Mason AC, Rambo RP, Bansbach CE, Badu-Nkansah A, Sirbu BM, Eichman BF, Cortez D. 2012. SMARCAL1 catalyzes fork regression and Holliday junction migration to maintain genome stability during DNA replication. Genes Dev 26: $151-162$.

Bétous R, Couch FB, Mason AC, Eichman BF, Manosas M, Cortez D. 2013. Substrate-selective repair and restart of replication forks by DNA translocases. Cell Rep 3: 1958-1969.

Bhowmick R, Minocherhomji S, Hickson ID. 2016. RAD52 facilitates mitotic DNA synthesis following replication stress. Mol Cell 64: 1117-1126.

Bouwman P, Aly A, Escandell JM, Pieterse M, Bartkova J, van der Gulden H, Hiddingh S, Thanasoula M, Kulkarni A, Yang Q, et al. 2010. 53BP1 loss rescues BRCA1 deficiency and is associated with triple-negative and BRCA-mutated breast cancers. Nat Struct Mol Biol 17: 688-695.

Bryant HE, Schultz N, Thomas HD, Parker KM, Flower D, Lopez E, Kyle S, Meuth M, Curtin NJ, Helleday T. 2005. Specific killing of BRCA2-deficient tumours with inhibitors of poly (ADP-ribose) polymerase. Nature 434: 913-917.

Ceccaldi R, Liu JC, Amunugama R, Hajdu I, Primack B, Petalcorin MI, O'Connor KW, Konstantinopoulos PA, Elledge SJ, Boulton SJ, et al. 2015. Homologous- recombination-deficient tumours are dependent on PolO-mediated repair. Nature 518: 258-262.

Chen CC, Feng W, Lim PX, Kass EM, Jasin M. 2018. Homology-directed repair and the role of BRCA1, BRCA2, and related proteins in genome integrity and cancer. Annu Rev Cancer Biol 2: 313-336.

Choi E, Park PG, Lee HO, Lee YK, Kang GH, Lee JW, Han W, Lee HC, Noh DY, Lekomtsev S, et al. 2012. BRCA2 fine-tunes the spindle assembly checkpoint through reinforcement of BubR1 acetylation. Dev Cell 22: 295-308.

Ciccia A, Nimonkar AV, Hu Y, Hajdu I, Achar YJ, Izhar L, Petit SA, Adamson B, Yoon JC, Kowalczykowski SC, et al. 2012. Polyubiquitinated PCNA recruits the ZRANB3 translocase to maintain genomic integrity after replication stress. Mol Cell 47: 396-409.

Cowell JK, LaDuca J, Rossi MR, Burkhardt T, Nowak NJ, Matsui S. 2005. Molecular characterization of the $t(3 ; 9)$ associated with immortalization in the MCF10A cell line. Cancer Genet Cytogenet 163: 23-29.

Cuella-Martin R, Oliveira C, Lockstone HE, Snellenberg S, Grolmusova N, Chapman JR. 2016. 53BP1 integrates DNA repair and 553 -dependent cell fate decisions via distinct mechanisms. Mol Cell 64: 51-64.

D'Andrea AD. 2010. Susceptibility pathways in Fanconi's anemia and breast cancer. N Engl J Med 362: 1909-1919.

Daniels MJ, Wang Y, Lee M, Venkitaraman AR. 2004. Abnormal cytokinesis in cells deficient in the breast cancer susceptibility protein BRCA2. Science 306: 876-879.

Davies H, Glodzik D, Morganella S, Yates LR, Staaf J, Zou X, Ramakrishna M, Martin S, Boyault S, Sieuwerts AM, et al. 2017. HRDetect is a predictor of BRCA1 and BRCA2 deficiency based on mutational signatures. Nat Med 23: 517-525.

Dehe PM, Gaillard PH. 2017. Control of structure-specific endonucleases to maintain genome stability. Nat Rev Mol Cell Biol 18: 315-330.

Ding X, Ray Chaudhuri A, Callen E, Pang Y, Biswas K, Klarmann KD, Martin BK, Burkett S, Cleveland L, Stauffer S, et al. 2016. Synthetic viability by BRCA2 and PARP1/ ARTD1 deficiencies. Nat Commun 7: 12425.
Dotsch V, Bernassola F, Coutandin D, Candi E, Melino G. 2010. p63 and p73, the ancestors of p53. Cold Spring Harb Perspect Biol 2: a004887.

Dungrawala H, Bhat KP, Le Meur R, Chazin WJ, Ding X, Sharan SK, Wessel SR, Sathe AA, Zhao R, Cortez D. 2017. RADX promotes genome stability and modulates chemosensitivity by regulating RAD51 at replication forks. Mol Cell 67: 374-386 e375.

Edwards SL, Brough R, Lord CJ, Natrajan R, Vatcheva R, Levine DA, Boyd J, Reis-Filho JS, Ashworth A. 2008. Resistance to therapy caused by intragenic deletion in BRCA2. Nature 451: 1111-1115.

Erkko H, Xia B, Nikkila J, Schleutker J, Syrjakoski K, Mannermaa A, Kallioniemi A, Pylkas K, Karppinen SM, Rapakko K, et al. 2007. A recurrent mutation in PALB2 in Finnish cancer families. Nature 446: 316-319.

Evers B, Jonkers J. 2006. Mouse models of BRCA1 and BRCA2 deficiency: Past lessons, current understanding and future prospects. Oncogene 25: 5885-5897.

Farmer H, McCabe N, Lord CJ, Tutt AN, Johnson DA, Richardson TB, Santarosa M, Dillon KJ, Hickson I, Knights C, et al. 2005. Targeting the DNA repair defect in BRCA mutant cells as a therapeutic strategy. Nature 434: 917-921.

Feng W, Jasin M. 2017. BRCA2 suppresses replication stressinduced mitotic and $\mathrm{G}_{1}$ abnormalities through homologous recombination. Nat Commun 8: 525 .

Feng Z, Scott SP, Bussen W, Sharma GG, Guo G, Pandita TK, Powell SN. 2011. Rad52 inactivation is synthetically lethal with BRCA2 deficiency. Proc Natl Acad Sci 108: 686-691.

Ganem NJ, Cornils H, Chiu SY, O'Rourke KP, Arnaud J, Yimlamai D, Thery M, Camargo FD, Pellman D. 2014. Cytokinesis failure triggers hippo tumor suppressor pathway activation. Cell 158: 833-848.

Greenblatt MS, Chappuis PO, Bond JP, Hamel N, Foulkes WD. 2001. TP53 mutations in breast cancer associated with BRCA1 or BRCA2 germ-line mutations: Distinctive spectrum and structural distribution. Cancer Res 61: 4092-4097.

Hanahan D, Weinberg RA. 2011. Hallmarks of cancer: The next generation. Cell 144: 646-674.

Higgs MR, Reynolds JJ, Winczura A, Blackford AN, Borel V, Miller ES, Zlatanou A, Nieminuszczy J, Ryan EL, Davies NJ, et al. 2015. BOD1L is required to suppress deleterious resection of stressed replication forks. Mol Cell 59: 462-477.

Iwabuchi K, Bartel PL, Li B, Marraccino R, Fields S. 1994. Two cellular proteins that bind to wild-type but not mutant p53. Proc Natl Acad Sci 91: 6098-6102.

Jensen RB, Carreira A, Kowalczykowski SC. 2010. Purified human BRCA2 stimulates RAD51-mediated recombination. Nature 467: 678-683.

Jonkers J, Meuwissen R, van der Gulden H, Peterse H, van der Valk M, Berns A. 2001. Synergistic tumor suppressor activity of BRCA2 and p53 in a conditional mouse model for breast cancer. Nat Genet 29: 418-425.

Kass EM, Lim PX, Helgadottir HR, Moynahan ME, Jasin M. 2016. Robust homology- directed repair within mouse mammary tissue is not specifically affected by Brca2 mutation. Nat Commun 7: 13241.

Kile AC, Chavez DA, Bacal J, Eldirany S, Korzhnev DM, Bezsonova I, Eichman BF, Cimprich KA. 2015. HLTF's ancient HIRAN domain binds 3 ' DNA ends to drive replication fork reversal. Mol Cell 58: 1090-1100.

Kolinjivadi AM, Sannino V, De Antoni A, Zadorozhny K, Kilkenny M, Techer H, Baldi G, Shen R, Ciccia A, Pellegrini L, et al. 2017. Smarcall-mediated fork reversal triggers Mre11dependent degradation of nascent DNA in the absence of Brca2 and stable Rad51 nucleofilaments. Mol Cell 67: 867881.e7.

Kondrashova O, Nguyen M, Shield-Artin K, Tinker AV, Teng NNH, Harrell MI, Kuiper MJ, Ho GY, Barker H, Jasin M, et al. 2017. Secondary somatic mutations restoring RAD51C and RAD51D associated with acquired resistance to the PARP inhibitor rucaparib in high-grade ovarian carcinoma. Cancer Discov 7: 984-998. 
Kuffer C, Kuznetsova AY, Storchova Z. 2013. Abnormal mitosis triggers p53- dependent cell cycle arrest in human tetraploid cells. Chromosoma 122: 305-318.

Kuznetsov SG, Liu P, Sharan SK. 2008. Mouse embryonic stem cell-based functional assay to evaluate mutations in BRCA2. Nat Med 14: 875-881.

Lai X, Broderick R, Bergoglio V, Zimmer J, Badie S, Niedzwiedz W, Hoffmann JS, Tarsounas M. 2017. MUS81 nuclease activity is essential for replication stress tolerance and chromosome segregation in BRCA2-deficient cells. Nat Commun 8: 15983

Laulier C, Cheng A, Stark JM. 2011. The relative efficiency of homology-directed repair has distinct effects on proper anaphase chromosome separation. Nucleic Acids Res 39: 5935-5944.

Lemacon D, Jackson J, Quinet A, Brickner JR, Li S, Yazinski S, You Z, Ira G, Zou L, Mosammaparast N, et al. 2017. MRE11 and EXO1 nucleases degrade reversed forks and elicit MUS81-dependent fork rescue in BRCA2-deficient cells. Nat Commun 8: 860.

Liu J, Doty T, Gibson B, Heyer WD. 2010. Human BRCA2 protein promotes RAD51 filament formation on RPA-covered single-stranded DNA. Nat Struct Mol Biol 17: 1260-1262.

Lok BH, Carley AC, Tchang B, Powell SN. 2013. RAD52 inactivation is synthetically lethal with deficiencies in BRCA1 and PALB2 in addition to BRCA2 through RAD51-mediated homologous recombination. Oncogene 32: 3552-3558.

Lord CJ, Ashworth A. 2016. BRCAness revisited. Nat Rev Cancer 16: 110-120.

Lord CJ, Ashworth A. 2017. PARP inhibitors: Synthetic lethality in the clinic. Science 355: 1152-1158.

Loveday C, Turnbull C, Ramsay E, Hughes D, Ruark E, Frankum JR, Bowden G, Kalmyrzaev B, Warren-Perry M, Snape K, et al. 2011. Germline mutations in RAD51D confer susceptibility to ovarian cancer. Nat Genet 43: 879-882.

Lukas C, Savic V, Bekker-Jensen S, Doil C, Neumann B, Pedersen RS, Grofte M, Chan KL, Hickson ID, Bartek J, et al. 2011. 53BP1 nuclear bodies form around DNA lesions generated by mitotic transmission of chromosomes under replication stress. Nat Cell Biol 13: 243-253.

Mateos-Gomez PA, Gong F, Nair N, Miller KM, Lazzerini-Denchi E, Sfeir A. 2015. Mammalian polymerase $\theta$ promotes alternative NHEJ and suppresses recombination. Nature 518: 254-257.

Maxwell KN, Wubbenhorst B, Wenz BM, De Sloover D, Pluta J, Emery L, Barrett A, Kraya AA, Anastopoulos IN, Yu S, et al. 2017. BRCA locus-specific loss of heterozygosity in germline BRCA1 and BRCA2 carriers. Nat Commun 8: 319.

Meindl A, Hellebrand H, Wiek C, Erven V, Wappenschmidt B, Niederacher D, Freund M, Lichtner P, Hartmann L, Schaal H, et al. 2010. Germline mutations in breast and ovarian cancer pedigrees establish RAD51C as a human cancer susceptibility gene. Nat Genet 42: 410-414.

Mijic S, Zellweger R, Chappidi N, Berti M, Jacobs K, Mutreja K, Ursich S, Ray Chaudhuri A, Nussenzweig A, Janscak P, et al. 2017. Replication fork reversal triggers fork degradation in BRCA2-defective cells. Nat Commun 8: 859.

Minocherhomji S, Ying S, Bjerregaard VA, Bursomanno S, Aleliunaite A, Wu W, Mankouri HW, Shen H, Liu Y, Hickson ID. 2015. Replication stress activates DNA repair synthesis in mitosis. Nature 528: 286-290.

Mondal G, Rowley M, Guidugli L, Wu J, Pankratz VS, Couch FJ. 2012. BRCA2 localization to the midbody by filamin A regulates cep55 signaling and completion of cytokinesis. Dev Cell 23: $137-152$.

Moynahan ME, Jasin M. 2010. Mitotic homologous recombination maintains genomic stability and suppresses tumorigenesis. Nat Rev Mol Cell Biol 11: 196-207.

Naim V, Wilhelm T, Debatisse M, Rosselli F. 2013. ERCC1 and MUS81-EME1 promote sister chromatid separation by processing late replication intermediates at common fragile sites during mitosis. Nat Cell Biol 15: 1008-1015.
Neelsen KJ, Lopes M. 2015. Replication fork reversal in eukaryotes: From dead end to dynamic response. Nat Rev Mol Cell Biol 16: 207-220.

Nik-Zainal S, Davies H, Staaf J, Ramakrishna M, Glodzik D, Zou X, Martincorena I, Alexandrov LB, Martin S, Wedge DC, et al. 2016. Landscape of somatic mutations in 560 breast cancer whole-genome sequences. Nature 534: 47-54.

Norquist B, Wurz KA, Pennil CC, Garcia R, Gross J, Sakai W, Karlan BY, Taniguchi T, Swisher EM. 2011. Secondary somatic mutations restoring BRCA1/2 predict chemotherapy resistance in hereditary ovarian carcinomas. J Clin Oncol 29: $3008-3015$

Patel KJ, Yu VP, Lee H, Corcoran A, Thistlethwaite FC, Evans MJ, Colledge WH, Friedman LS, Ponder BA, Venkitaraman AR. 1998. Involvement of Brca2 in DNA repair. Mol Cell 1: 347-357.

Pedersen RS, Karemore G, Gudjonsson T, Rask MB, Neumann B, Heriche JK, Pepperkok R, Ellenberg J, Gerlich DW, Lukas $\mathrm{J}$, et al. 2016. Profiling DNA damage response following mitotic perturbations. Nat Commun 7: 13887.

Pennington KP, Walsh T, Harrell MI, Lee MK, Pennil CC, Rendi $\mathrm{MH}$, Thornton A, Norquist BM, Casadei S, Nord AS, et al. 2014. Germline and somatic mutations in homologous recombination genes predict platinum response and survival in ovarian, fallopian tube, and peritoneal carcinomas. Clin Cancer Res 20: 764-775.

Polak P, Kim J, Braunstein LZ, Karlic R, Haradhavala NJ, Tiao G, Rosebrock D, Livitz D, Kubler K, Mouw KW, et al. 2017. A mutational signature reveals alterations underlying deficient homologous recombination repair in breast cancer. Nat Genet 49: $1476-1486$.

Prakash R, Zhang Y, Feng W, Jasin M. 2015. Homologous recombination and human health: The roles of BRCA1, BRCA2, and associated proteins. Cold Spring Harb Perspect Biol 7: a016600.

Quigley D, Alumkal JJ, Wyatt AW, Kothari V, Foye A, Lloyd P, Aggarwal R, Kim W, Lu E, Schwartzman J, et al. 2017. Analysis of circulating cell-free DNA identifies multiclonal heterogeneity of BRCA2 reversion mutations associated with resistance to PARP inhibitors. Cancer Discov 7: 999-1005.

Quinet A, Lemacon D, Vindigni A. 2017. Replication fork reversal: Players and guardians. Mol Cell 68: 830-833.

Rahman N, Seal S, Thompson D, Kelly P, Renwick A, Elliott A, Reid S, Spanova K, Barfoot R, Chagtai T, et al. 2007. PALB2, which encodes a BRCA2-interacting protein, is a breast cancer susceptibility gene. Nat Genet 39: 165-167.

Ramus SJ, Bobrow LG, Pharoah PD, Finnigan DS, Fishman A, Altaras M, Harrington PA, Gayther SA, Ponder BA, Friedman LS. 1999. Increased frequency of TP53 mutations in BRCA1 and BRCA2 ovarian tumours. Genes Chromosomes Cancer 25: 91-96.

Ray Chaudhuri A, Callen E, Ding X, Gogola E, Duarte AA, Lee JE, Wong N, Lafarga V, Calvo JA, Panzarino NJ, et al. 2016. Replication fork stability confers chemoresistance in BRCAdeficient cells. Nature 535: 382-387.

Robinson D, Van Allen EM, Wu YM, Schultz N, Lonigro RJ, Mosquera JM, Montgomery B, Taplin ME, Pritchard CC, Attard G, et al. 2015. Integrative clinical genomics of advanced prostate cancer. Cell 161: 1215-1228.

Rondinelli B, Gogola E, Yucel H, Duarte AA, van de Ven M, van der Sluijs R, Konstantinopoulos PA, Jonkers J, Ceccaldi R, Rottenberg S, et al. 2017. EZH2 promotes degradation of stalled replication forks by recruiting MUS81 through histone H3 trimethylation. Nat Cell Biol 19: 1371-1378.

Roy R, Chun J, Powell SN. 2011. BRCA1 and BRCA2: Different roles in a common pathway of genome protection. Nat Rev Cancer 12: 68-78.

Sakai W, Swisher EM, Karlan BY, Agarwal MK, Higgins J, Friedman C, Villegas E, Jacquemont C, Farrugia DJ, Couch FJ, et al. 2008. Secondary mutations as a mechanism of cisplatin resistance in BRCA2-mutated cancers. Nature 451: $1116-1120$ 
Schlacher K, Christ N, Siaud N, Egashira A, Wu H, Jasin M. 2011. Double-strand break repair-independent role for BRCA2 in blocking stalled replication fork degradation by MRE11. Cell 145: 529-542.

Schlacher K, Wu H, Jasin M. 2012. A distinct replication fork protection pathway connects Fanconi anemia tumor suppressors to RAD51-BRCA1/2. Cancer Cell 22: 106-116.

Schoonen PM, Talens F, Stok C, Gogola E, Heijink AM, Bouwman P, Foijer F, Tarsounas M, Blatter S, Jonkers J, et al. 2017. Progression through mitosis promotes PARP inhibitor-induced cytotoxicity in homologous recombination-deficient cancer cells. Nat Commun 8: 15981.

Somyajit K, Saxena S, Babu S, Mishra A, Nagaraju G. 2015. Mammalian RAD51 paralogs protect nascent DNA at stalled forks and mediate replication restart. Nucleic Acids Res 43: 9835-9855.

Soule HD, Maloney TM, Wolman SR, Peterson WD Jr, Brenz R, McGrath CM, Russo J, Pauley RJ, Jones RF, Brooks SC. 1990. Isolation and characterization of a spontaneously immortalized human breast epithelial cell line, MCF-10. Cancer Res 50: 6075-6086.

Taglialatela A, Alvarez S, Leuzzi G, Sannino V, Ranjha L, Huang JW, Madubata C, Anand R, Levy B, Rabadan R, et al. 2017. Restoration of replication fork stability in BRCA1- and BRCA2-deficient cells by inactivation of SNF2-family fork remodelers. Mol Cell 68: 414-430 e418.

Thangavel S, Berti M, Levikova M, Pinto C, Gomathinayagam S, Vujanovic M, Zellweger R, Moore H, Lee EH, Hendrickson EA, et al. 2015. DNA2 drives processing and restart of reversed replication forks in human cells. J Cell Biol 208: 545-562.

Thorslund T, McIlwraith MJ, Compton SA, Lekomtsev S, Petronczki M, Griffith JD, West SC. 2010. The breast cancer tumor suppressor BRCA2 promotes the specific targeting of RAD51 to single-stranded DNA. Nat Struct Mol Biol 17: 1263-1265.
Tischkowitz M, Xia B, Sabbaghian N, Reis-Filho JS, Hamel N, Li G, van Beers EH, Li L, Khalil T, Quenneville LA, et al. 2007. Analysis of PALB2/FANCN-associated breast cancer families. Proc Natl Acad Sci 104: 6788-6793.

Tutt A, Gabriel A, Bertwistle D, Connor F, Paterson H, Peacock J, Ross G, Ashworth A. 1999. Absence of Brca2 causes genome instability by chromosome breakage and loss associated with centrosome amplification. Curr Biol 9: 1107-1110.

Wang AT, Kim T, Wagner JE, Conti BA, Lach FP, Huang AL, Molina H, Sanborn EM, Zierhut H, Cornes BK, et al. 2015. A dominant mutation in human RAD51 reveals its function in DNA interstrand crosslink repair independent of homologous recombination. Mol Cell 59: 478-490.

Wang Q, Zou Y, Nowotschin S, Kim SY, Li QV, Soh CL, Su J, Zhang C, Shu W, Xi Q, et al. 2017. The p53 family coordinates Wnt and nodal inputs in mesendodermal differentiation of embryonic stem cells. Cell Stem Cell 20: 70-86.

Wooster R, Bignell G, Lancaster J, Swift S, Seal S, Mangion J, Collins N, Gregory S, Gumbs C, Micklem G. 1995. Identification of the breast cancer susceptibility gene BRCA2. Nature 378: 789-792.

Xu S, Wu X, Wu L, Castillo A, Liu J, Atkinson E, Paul A, Su D, Schlacher K, Komatsu Y, et al. 2017a. Abro1 maintains genome stability and limits replication stress by protecting replication fork stability. Genes Dev 31: 1469-1482.

Xu Y, Ning S, Wei Z, Xu R, Xu X, Xing M, Guo R, Xu D. 2017 b. 53BP1 and BRCA1 control pathway choice for stalled replication restart. Elife 6: e30523.

Ying S, Minocherhomji S, Chan KL, Palmai-Pallag T, Chu WK, Wass T, Mankouri HW, Liu Y, Hickson ID. 2013. MUS81 promotes common fragile site expression. Nat Cell Biol 15: 1001-1007.

Zellweger R, Dalcher D, Mutreja K, Berti M, Schmid JA, Herrador R, Vindigni A, Lopes M. 2015. Rad51-mediated replication fork reversal is a global response to genotoxic treatments in human cells. J Cell Biol 208: 563-579. 


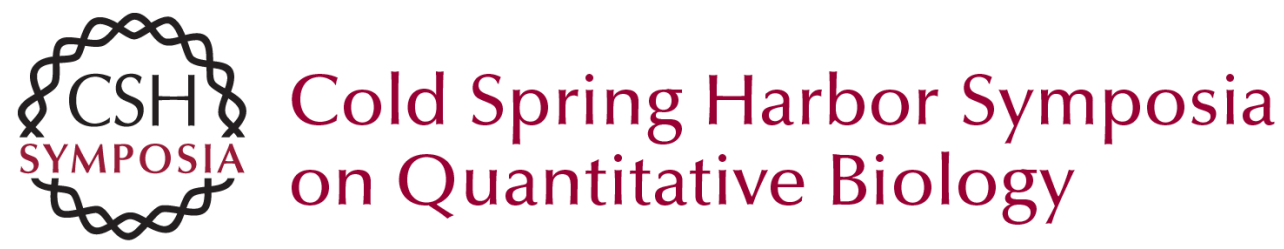

\section{Homologous Recombination and Replication Fork Protection: BRCA2 and More!}

Weiran Feng and Maria Jasin

Cold Spring Harb Symp Quant Biol 2017 82: 329-338 originally published online April 23, 2018 Access the most recent version at doi:10.1101/sqb.2017.82.035006

References This article cites 92 articles, 17 of which can be accessed free at: http://symposium.cshlp.org/content/82/329.full.html\#ref-list-1

Creative This article is distributed under the terms of the

Commons http://creativecommons.org/licenses/by/4.0/, which permits unrestricted

License reuse and redistribution provided that the original author and source are credited.

Email Alerting Receive free email alerts when new articles cite this article - sign up in Service the box at the top right corner of the article or click here. 